\title{
Drivers can poorly predict their own driving impairment: a comparison between measurements of subjective and objective driving quality
}

\author{
Joris C. Verster • Thomas Roth
}

Received: 4 April 2011 / Accepted: 24 June 2011 /Published online: 14 July 2011

(C) The Author(s) 2011. This article is published with open access at Springerlink.com

\begin{abstract}
Rationale Adverse drug effects such as reduced alertness may cause drivers to be unaware that their driving is impaired. Objective This study was conducted to examine if drivers are adequately aware of their driving ability when treated with central nervous system drugs.

Methods Data from three clinical trials applying the on-theroad driving test were used to compare the primary outcome measure, the Standard Deviation of Lateral Position, with subjective assessments of alertness before driving, as well as perceived driving quality, and mental effort to perform the test.

Results The analyses revealed significant correlations for perceived driving quality $(r=-0.498, p<0.0001)$, mental effort to perform the test $(r=0.408, p<0.0001)$, and alertness taken before driving $(r=-0.115, p<0.017)$. The predictive validity $\left(R^{2}\right)$ of perceived driving quality $(24.8 \%)$, mental effort (16.6\%), and alertness before driving $(1.3 \%)$ was low.

Conclusion The analyses show that subjective assessments do not robustly relate to actual driving performance either in terms of judgments about alertness before the drive or ratings of performance after the drive.
\end{abstract}

Keywords Driving · SDLP. Subjective assessments · Alertness $\cdot$ Effort

\section{J. C. Verster $(\bowtie)$}

Utrecht Institute for Pharmaceutical Sciences,

Division of Pharmacology, Utrecht University,

Universiteitsweg 99,

3584 CG Utrecht, The Netherlands

e-mail: j.c.verster@uu.nl

T. Roth

Sleep Disorders and Research Center, Henry Ford Health System,

Detroit, MI, USA

\section{Introduction}

Drivers who experience reduced alertness or other adverse effects of central nervous system (CNS) drugs often show impaired driving. Being aware of adverse CNS drug effects and recognizing that driving is impaired are essential for making appropriate countermeasures. If individuals are aware of adverse drug effects, they may choose not to drive. Alternatively, if they acknowledge that their driving is impaired, they can stop driving before an accident occurs or can drive shorter periods of time, or avoid taking the drug on future occasions when they plan to drive. Unfortunately, research has shown that drivers can poorly judge their own driving performance.

Drivers often overestimate their skills and abilities and do not recognize impairment. For example, it has been shown that $70-90 \%$ of drivers feel their driving is better and less risky than that of the average driver (Svenson 1978, 1981; McCormick et al. 1986; Groeger and Brown 1989; Sivak et al. 1989). Men and women do not differ in overestimating their own driving ability (McCormick et al. 1986; Groeger and Brown 1989). Subjective ratings of driving ability were shown to differ between countries and cultures and may depend on factors such as driving experience, age, personality, and crash experience (Matthews and Moran 1986; Sivak et al. 1989; Holland 1993; Lajunen and Summala 1995; Lajunen et al. 1998). Although there is a bias towards a more positive view of drivers' own skills and abilities, this does not necessarily mean that they are unable to adequately assess changes in their driving performance, for example those induced by CNS drugs and alcohol. Research that tested drivers who were unfit for driving due to medical conditions such as stroke showed that patients who fail a driving test often have limited awareness of their disability and corresponding 
negative consequences with regard to driving (Patomella et al. 2008). Of 152 elderly drivers tested in a driving simulator, $65 \%$ rated their performance better than the average driver (Freund et al. 2005). However, about half of them showed unsafe driving performance on the simulator test.

Previous research that compared subjective driving quality and actual driving performance suffers from methodological issues. The measurement of actual driving performance was typically accomplished by means of psychometric tests in the laboratory, driving simulators, or closed-road driving tests. It can be questioned to what extent these tests represent and predict actual driving and the degree to which subjects think this relates to real driving. The primary reason for this is that in these tests there is no risk to the driver, which will cause participants to behave differently than in actual traffic. Other studies did use on-road assessments, but performance was rated by the experimenter or a driving instructor, not the driver. Finally, measurement tools are not standardized across studies which make comparing the results difficult.

This paper examines the relationship between subjective assessments and actual driving impairment, objectively measured by the on-the-road driving test. The on-the-road driving test in normal traffic (Verster and Roth 2011) is the gold standard method to examine the effects of CNS drugs on driving. The 100-km standardized test was developed in the 1980s (O'Hanlon et al. 1982; O'Hanlon 1984) and has been applied in over 75 clinical trials. Participants are instructed to drive with a steady lane position and a constant speed. The Standard Deviation of Lateral Position (SDLP), i.e., the "weaving" of the car, is the primary parameter of the test. The test has proven sensitivity to measure dose-dependent impairment of a variety of CNS drugs, and comparative research with alcohol, THC, and benzodiazepines showed that doserelated changes in SDLP correlate high with the risk of becoming involved in traffic accidents (Owens and Ramaekers 2009).

As a standard procedure in clinical trials applying the onthe-road driving test, subjective assessments are conducted to determine levels of alertness before driving, and after driving perceived driving quality, and mental effort needed to perform the drive. Based on the literature discussed above, it is hypothesized that drivers are unable to accurately estimate (changes in) their driving performance.

\section{Methods}

Data from three studies were used to compose the current data set (Verster et al. 2002a, b, 2003, 2006). The Medical Ethics Committee of the University Medical Center Utrecht approved the study protocols, and subjects were treated according to ICH guidelines for Good Clinical Practice and the Declaration of Helsinki and its amendments.

\section{Participants}

A total of 96 healthy volunteers completed the three studies. In each study, male and female participants were equally represented. Mean (SD) age was 23.9 (2.2) years old. Written informed consent was obtained before their participation. Subjects were medically screened, used no concomitant medication other than paracetamol and oral contraceptives, and they had no unstable medical disorders, history of psychiatric disease, alcohol or drug abuse. Before the start and at the end of the studies, blood chemistry and hematology and urinalysis were determined, and a 12-lead ECG was recorded. All assessments were within normal limits. To confirm drug status, at all visits subjects were tested on the presence of alcohol and drugs of abuse (amphetamines, barbiturates, cannabinoids, benzodiazepines, cocaine, and opioids). In addition, female subjects underwent $\beta$-HCG pregnancy tests. None of the subjects were positive on any of these drugs. Subjects possessed a valid driver's license, drove more than 5,000 km/year during each of the past 3 years, and were trained on the driving tests. A thorough discussion on the inclusion and exclusion criteria of participants and description of the study designs can be found in the individual reports (Verster et al. 2002a, b, 2003, 2006).

\section{Treatments}

A variety of treatments were tested in the three studies, including alcohol, hypnotics, anxiolytics, analgesics, and $\mathrm{H}_{1}$-antihistamines. The specific drugs and their dosages are summarized in Table 1.

\section{The on-the-road driving test}

Standardized 100-km driving tests (Verster and Roth, 2011) were performed on a primary highway during normal traffic, between the cities of Utrecht and Arnhem. A camera, mounted on the roof of the test vehicle, measured the vehicle's lateral position relative to the road delineation. Participants were instructed to drive with a steady lateral position within the right traffic lane while maintaining a constant speed of $95 \mathrm{~km} / \mathrm{h}(60 \mathrm{mph})$. The amount of "weaving" of the car, measured by the standard deviation of the lateral position (SDLP, centimeters), was the primary outcome parameter. The standard deviation of speed (kilometers per hour) was a secondary parameter. Duration of the driving test was approximately $75 \mathrm{~min}$.

Patients were allowed to deviate from the instructions to overtake a slower-moving vehicle in the same traffic lane. 
Table 1 Administered drugs in the three driving studies

\begin{tabular}{|c|c|c|c|}
\hline Reference & Subjects & $\begin{array}{l}\text { Time after } \\
\text { administration }(\mathrm{h})\end{array}$ & Treatments \\
\hline (Verster et al. 2002a) & 30 & 1 & $\begin{array}{l}\text { Placebo } \\
\text { Alcohol (BAC 0.05\%) }\end{array}$ \\
\hline (Verster et al. 2002a) & 30 & $9-10$ & $\begin{array}{l}\text { Placebo } \\
\text { Zaleplon } 10 \mathrm{mg} \\
\text { Zaleplon } 20 \mathrm{mg} \\
\text { Zolpidem } 10 \mathrm{mg} \\
\text { Zolpidem } 20 \mathrm{mg}\end{array}$ \\
\hline $\begin{array}{r}\text { (Verster et al. 2002b; } \\
\text { Verster et al. 2006) }\end{array}$ & 18 & 1 & $\begin{array}{l}\text { Placebo } \\
\text { Bromfenac } 25 \mathrm{mg} \\
\text { Bromfenac } 50 \mathrm{mg} \\
\text { Oxycodone/paracetamol 5/325 mg } \\
\text { Oxycodone/paracetamol 10/650 mg } \\
\text { Alprazolam } 1 \mathrm{mg}\end{array}$ \\
\hline (Verster et al. 2003) & 48 & 1 (day 1 and day 4$)$ & $\begin{array}{l}\text { Placebo } \\
\text { Levocetirizine } 5 \mathrm{mg} \\
\text { Diphenhydramine } 50 \mathrm{mg}\end{array}$ \\
\hline
\end{tabular}

$B A C$ blood alcohol concentration

subject estimates of their driving quality and mental effort to perform it were measured. Participants indicated the perceived quality of their driving performance on a visual analog scale, which ranged from 0 ("I drove exceptionally poorly") to 20 ("I drove exceptionally well") around a midpoint of "I drove normally." The level of mental effort they had to exert to perform the task was indicated on a $15-\mathrm{cm}$ interval scale, ranging from "absolutely no effort" to "extreme effort" (Zijlstra and Van Doorn 1985; Meijman et al. 1986).

Statistical analysis

For each treatment, difference scores from placebo were calculated for each dependent variable. The data of the

Before the driving test, subjective alertness was measured using a 21-point visual analog scale. After the driving test,

Table 2 Differences from placebo (centimeters) for SDLP, perceived driving quality, mental effort to perform the test, and alertness before driving

${ }^{*} p<0.05$, significant differences

\begin{tabular}{lccccc}
\hline Treatment & $N$ & SDLP & Mental effort & Driving quality & Alertness \\
\hline Zolpidem (20 mg) & 30 & $+10.41^{*}$ & $+2.25^{*}$ & $-4.23^{*}$ & $-5.53^{*}$ \\
Alprazolam (1 mg) & 18 & $+9.62^{*}$ & $+3.76^{*}$ & $-5.17^{*}$ & $+3.94^{*}$ \\
Zolpidem (10 mg) & 30 & $+3.35^{*}$ & +0.52 & -1.75 & $-2.83^{*}$ \\
Diphenhydramine (50 mg, day 1) & 48 & $+2.74^{*}$ & $+2.67^{*}$ & $-3.66^{*}$ & $+2.94^{*}$ \\
Oxycodone/paracetamol (10/650 mg) & 18 & +1.87 & $+3.29^{*}$ & $-3.83^{*}$ & $+4.22^{*}$ \\
Diphenhydramine (50 mg, day 4) & 48 & $+1.56^{*}$ & +0.42 & -0.44 & $+1.46^{*}$ \\
Alcohol (BAC=0.05\%) & 29 & $+1.03^{*}$ & +0.07 & -0.12 & $-3.33^{*}$ \\
Bromfenac (50 mg) & 18 & +0.51 & +0.59 & -1.62 & +0.61 \\
Levocetirizine (5 mg, day 4) & 48 & +0.46 & -0.04 & -0.22 & +0.02 \\
Zaleplon (10 mg) & 30 & -0.45 & -0.07 & +0.59 & -0.30 \\
Zaleplon (20 mg) & 30 & +0.43 & +0.84 & -0.26 & -1.30 \\
Levocetirizine (5 mg, day 1) & 48 & +0.22 & +0.85 & -1.23 & +0.06 \\
Oxycodone/paracetamol (5/325 mg) & 18 & -0.65 & +0.93 & -1.53 & $+1.94 *$ \\
Bromfenac (25 mg) & 18 & -0.66 & +0.42 & -1.82 & +0.61 \\
\hline
\end{tabular}


Fig. 1 Relationship between changes from placebo of SDLP and subjective driving ability

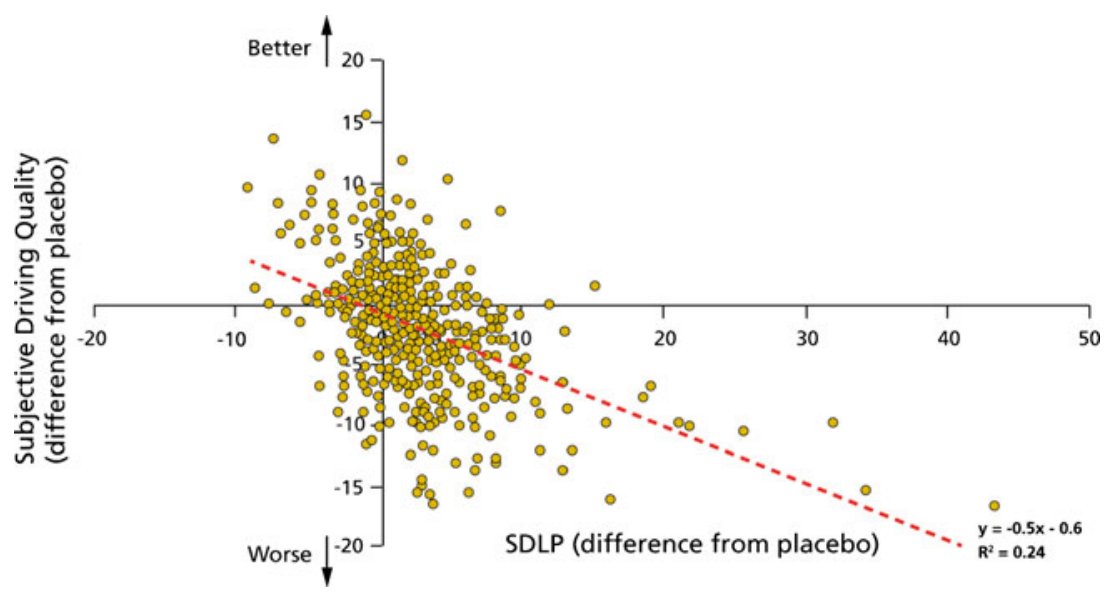

(24.8\%) and mental effort (16.6\%) was moderate to low, but importantly, predictive validity of alertness before driving $(1.3 \%)$ was virtually absent. Thus, the only measure taken before the actual drive, and therefore the only measure of predicting rather than evaluating performance, showed the lowest predictive validity. Overall results and those for individual drugs are summarized in Table 3.

It is evident from Table 3 (data from individual drug treatment analyses) that the correlation between SDLP differences from placebo and subjective assessments is highly variable between studies with no clear relation with drugs, doses, or times of drug administration relative to time of driving. It is important to recognize that these are the data that are gathered for individual research projects.

\section{Discussion}

The analyses show that subjective assessments, especially predictive ones, do not robustly relate to actual driving performance either in terms of judgments about alertness before the drive or ratings of performance after the drive. This is especially true when the data are analyzed study by study. The present findings have important clinical and public health
Fig. 2 Relationship between changes from placebo of SDLP and mental effort to perform the driving test

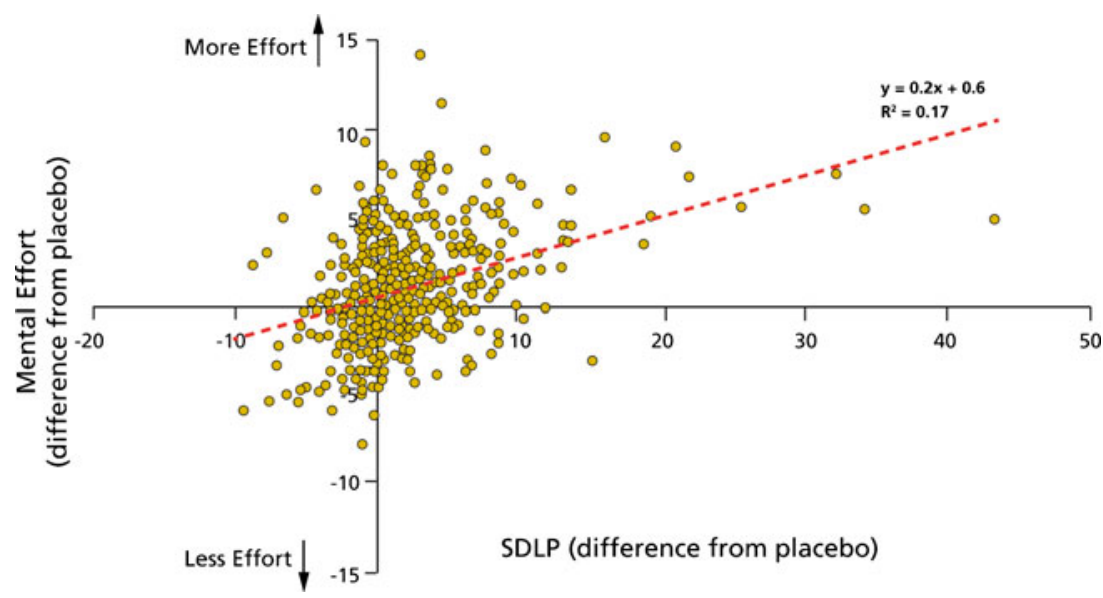


Fig. 3 Relationship between changes from placebo of SDLP and alertness before the driving test

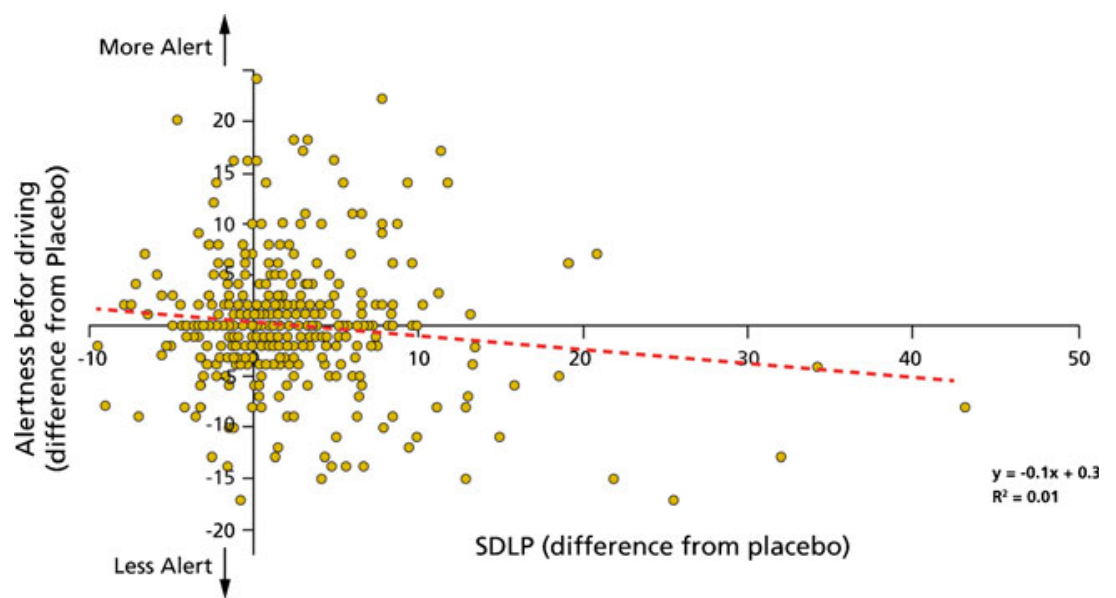

implications as it is typically the patient's responsibility to make a decision whether or not to initiate a drive and whether to continue driving after they have experienced how they drive under the medication. In fact, the direction to patients to experience the drug several times before they undertake operating machinery presupposes that patients will accurately assess their performance under the influence of the medication and thereby make informed decisions.

Whenever participants drive a vehicle, they make a subjective estimate of how they are feeling at that time and location in comparison to their normal driving state. Therefore, the rationale for the statistical analyses was to use difference scores from placebo. This approach was chosen because it provides information if drivers can adequately assess changes from their normal driving performance. Where there are significant differences from placebo for SDLP (see Table 3), these are associated with increases in mental effort (except for alcohol) and decreases in perceived driving quality. Therefore, participants were retrospectively consistently aware of impairment. These findings were much less consistent for differences in SDLP and alertness assessed before driving. For example, while both drugs significantly impair driving, alertness before the test was significantly decreased for zolpidem, but increased for diphenhydramine (day 4). The analyses on difference scores from placebo revealed statistically significant correlations for perceived driving quality, mental effort to perform the test, and alertness before driving, but the percentage of predictive validity towards changes in SDLP was low. Correlations between absolute values of SDLP and subjective assessments of driving ability are not very informative. The primary reason for this is that SDLP

Table 3 Correlation between SDLP differences from placebo (centimeters) and perceived driving quality, mental effort to perform the test, and alertness before driving

\begin{tabular}{|c|c|c|c|c|c|}
\hline Treatment & $N$ & $\triangle \mathrm{SDLP}$ & Mental effort & Driving quality & Alertness \\
\hline Zolpidem (20 mg) & 30 & $+10.41 * * *$ & $+0.488 * *$ & $-0.809 * *$ & $-0.480 * *$ \\
\hline Alprazolam (1 mg) & 18 & $+9.62 * * *$ & +0.364 & -0.205 & +0.366 \\
\hline Zolpidem (10 mg) & 30 & $+3.35^{* * *}$ & $+0.512 * *$ & $-0.592 * *$ & $-0.653 * *$ \\
\hline Diphenhydramine (50 mg, day 1 ) & 48 & $+2.74 * * *$ & +0.160 & $-0.438 * *$ & +0.169 \\
\hline Oxycodone/paracetamol (10/650 mg) & 18 & +1.87 & +0.455 & $-0.476 *$ & -0.189 \\
\hline Diphenhydramine (50 mg, day 4) & 48 & $+1.56^{* * *}$ & +0.268 & $-0.526^{* *}$ & $+0.327^{*}$ \\
\hline Alcohol $(\mathrm{BAC}=0.05 \%)$ & 29 & $+1.03 * * *$ & -0.002 & -0.153 & +0.148 \\
\hline Bromfenac $(50 \mathrm{mg})$ & 18 & +0.51 & +0.308 & -0.334 & +0.070 \\
\hline Levocetirizine ( $5 \mathrm{mg}$, day 4 ) & 48 & +0.46 & $+0.378 * *$ & $-0.319 *$ & +0.126 \\
\hline Zaleplon (10 mg) & 30 & -0.45 & $+0.381 *$ & -0.305 & -0.278 \\
\hline Zaleplon (20 mg) & 30 & +0.43 & $+0.447 *$ & $-0.515 * *$ & $-0.409 *$ \\
\hline Levocetirizine (5 mg, day 1 ) & 48 & +0.22 & $+0.361 *$ & $-0.347 *$ & -0.068 \\
\hline Oxycodone/paracetamol (5/325 mg) & 18 & -0.65 & $+0.514 *$ & $-0.513 *$ & +0.251 \\
\hline Bromfenac (25 mg) & 18 & -0.66 & $+0.562 *$ & $-0.541 *$ & $+0.608 * *$ \\
\hline Overall & 431 & +2.03 & $0.408 * *$ & $-0.498 * *$ & $-0.115 *$ \\
\hline
\end{tabular}

$* p<0.05$ and $* * p<0.01$, significance of the Pearson's $r$ correlation with SDLP; $* * * p<0.05$, SDLP differs significantly from placebo 
values differ greatly between participants (Verster and Roth 2011). Hence, one subject may regard an SDLP of $15 \mathrm{~cm}$ as normal, whereas another participant regards an SDLP value of $25 \mathrm{~cm}$ as normal. As placebo (absolute) values between participants are so variable, they cannot be used to provide a quantitative comparison.

These data show that participants are not always able to accurately judge changes in their driving performance after administration of CNS drugs. The data also show that mental effort to conduct the test is a poor indicator of the driving impairment they experienced. Of greater concern is that the level of alertness before driving does not predict driving performance. Although the correlation was significant (at least in part due to the large number of subjects as well as the number of different drug treatments), the predictive validity was only $1.3 \%$. The poor correlation between perceived level of alertness and actual performance is caused by the fact that the awareness of reduced alertness differs between CNS drugs (See Table 3). For example, whereas driving was severely impaired after both administration of zolpidem $(20 \mathrm{mg})$ and alprazolam $(1 \mathrm{mg})$, alertness correlated significantly with SDLP only after zolpidem, but not after alprazolam. Also, perceived driving quality did not correlate significantly with driving impairment caused by alprazolam. In contrast, after administration of zolpidem even at a high dose $(20 \mathrm{mg})$, drivers were fully aware of their performance impairment. These findings illustrate the importance of obtaining these subjective assessments to compliment the objective measurements of driving performance. It provides useful information whether drivers are aware of driving impairment, or if participants have to invest significantly more effort to prevent driving impairment.

A limitation of this study is that we did not directly ask participants before the start of the driving test to what extent they anticipated their driving would be impaired. Nevertheless, it can be assumed that many people make this judgment based on the level of alertness they experience. Of interest for future research is to conduct assessments of subjective driving quality during the driving test. The objective and subjective measurements would then be truly concurrent assessments. Making these assessments several times during the driving test would also enable showing whether the subjective assessments are in line with the objective performance decrement generally seen during the driving test (Verster and Roth 2011).

The results of the current analyses are consistent with the literature on sleep deprivation that also shows a lack of a relation between perceived performance impairment and actual performance impairment (Herscovitch and Broughton 1981; Van Dongen and Belenky 2009). This has important clinically relevant implications. First, people should not rely solely on their perceived alertness when deciding whether or not it is safe to drive a car after using CNS drugs. The poor correlation between subjective and objective driving ability shows that the general advice that "patients should listen to their body, and not drive if they feel their driving is impaired" should not be relied on because patients may not be aware of their driving impairment. Physicians should therefore urge patients to follow-up labeling instructions regarding drug effects on driving. In addition, patients should be informed that they may not be aware of impaired driving, or do not experience reduced alertness while at the same time their performance may be significantly impaired. Physicians should take a more active role in educating patients about the possible risks of drug use in combination with daily activities such as driving. This proactive approach by physicians is important because many patients do not read nor followup instructions of prescription drug labeling (Veldhuijzen et al. 2006). Although several CNS drugs can impair driving, often alternative pharmacological treatments are available that do not affect driving ability or more typically have a lesser effect on driving. When a patient needs to drive a car regularly, physicians should incorporate the data on the effects of the drug on driving performance into their overall selection of the most appropriate medication for this patient.

Acknowledgment The studies presented in this article were financially supported by Wyeth-Ayerst Research and UCB Pharma.

Conflicts of interest The authors have no conflicts of interest to disclose.

Open Access This article is distributed under the terms of the Creative Commons Attribution Noncommercial License which permits any noncommercial use, distribution, and reproduction in any medium, provided the original author(s) and source are credited.

\section{References}

Freund B, Colgrove LA, Burke BL, McLoad R (2005) Self-rated performance among elderly drivers referred for driving evaluation. Accid Anal Prev 37:613-618

Groeger JA, Brown D (1989) Assessing one's own and others' driving ability: influences of sex, age and experience. Accid Anal Prev $21: 155-168$

Herscovitch J, Broughton R (1981) Sensitivity of the Stanford Sleepiness Scale to the effects of cumulative partial sleep deprivation and recovery oversleeping. Sleep 4:83-91

Holland CA (1993) Self-bias in older drivers' judgments of accident likelihood. Accid Anal Prev 25:431-441

Lajunen T, Summala H (1995) Driving experience, personality, and skill and safety-motive dimensions in drivers' self-assessments. Person Individ Diff 19:307-318

Lajunen T, Corry A, Summala H, Hartley L (1998) Cross-cultural differences in drivers' self-assessments of their perceptual-motor and safety skills: Australian and Finns. Person Individ Diff 24:539-550 
Matthews ML, Moran AR (1986) Age differences in male drivers' perception of accident risk: the role of perceived driving ability. Accid Anal Prev 18:299-313

McCormick IA, Walkey FH, Green DE (1986) Comparative perceptions of driver ability - a confirmation and expansion. Accid Anal Prev 18:205-208

Meijman TF, Zijlstra FRH, Kompier MAJ, Mulders HPG, Broerse JPJ (1986) The measurement of perceived effort. Report. University of Groningen, Department of Occupational Psychology, Biological Center, Haren, The Netherlands

O'Hanlon JF (1984) Driving under the influence of drugs: rationale for, and application of, a new test. Br J Clin Pharmacol 18:121S-129S

O'Hanlon JF, Haak TW, Blaauw GJ, Riemersma JBJ (1982) Diazepam impairs lateral position control in highway driving. Science 217:79-81

Owens K, Ramaekers JG (2009) Drugs, driving, and models to measure driving impairment. In: Verster JC, Pandi-Perumal SR, Ramaekers JG, de Gier JJ (eds) Drugs, driving and traffic safety. Birkhauser Verlag AG, Basel, pp 43-58

Patomella AH, Kottorp A, Tham K (2008) Awareness of driving disability in people with stroke tested in a simulator. Scand J Occup Ther 15:184-192

Sivak M, Soler J, Tränkle U (1989) Cross-cultural differences in driver self-assessment. Accid Anal Prev 21:371-375

Svenson O (1978) Risks of road transportation in a psychological perspective. Accid Anal Prev 10:267-280

Svenson O (1981) Are we all less risky and more skillful than our fellow drivers? Acta Psychol 47:143-148

Van Dongen HP, Belenky G (2009) Individual differences in vulnerability to sleep loss in the work environment. Ind Health 47:518-526
Veldhuijzen DS, Van Wijck AJM, Verster JC, Kalkman CJ, Kenemans JL, Olivier B, Volkerts ER (2006) The impact of patients' psychotropic drug knowledge and warning labels on the decision whether to drive a car or not. Traffic Inj Prev 7:360-364

Verster JC, Roth T (2011) Standard operation procedures for conducting the on-the-road driving test, and measurement of the standard deviation of lateral position (SDLP). Int J Gen Med 4:359-371

Verster JC, Volkerts ER, Schreuder AHCML, Eijken EJE, Van Heuckelum JHG, Veldhuijzen DS, Verbaten MN, Patat A, Paty I (2002a) Residual effects of middle-of-the-night administration of zaleplon and zolpidem on driving ability, memory functions and psychomotor performance. J Clin Psychopharmacol 22:576583

Verster JC, Volkerts ER, Verbaten MN (2002b) Effects of alprazolam on driving ability, memory functioning and psychomotor performance: a randomized, placebo-controlled study. Neuropsychopharmacol 27:260-269

Verster JC, De Weert AM, Bijtjes SIR, Aarab M, van Oosterwijck AWAA, Eijken EJE, Verbaten MN, Volkerts ER (2003) Driving ability after acute and subchronic administration of levocetirizine and diphenhydramine: a randomized, double blind, placebocontrolled trial. Psychopharmacol 169:84-90

Verster JC, Veldhuijzen DS, Volkerts ER (2006) Effects of an opioid (oxycodone/paracetamol) and an NSAID (bromfenac) on driving ability, memory functioning, psychomotor performance, pupil size and mood. Clin J Pain 22:499-504

Zijlstra FRH, van Doorn L (1985) The construction of a scale to measure perceived effort. Report Delft University of Technology, Delft, The Netherlands 